\title{
Pharmacokinetics and bioavailability of midazolam in rats following single dose administration of intravenous solution, intranasal dispersion, and in situ nasal gel
}

\author{
Elahehnaz Parhizkar ${ }^{1}$, Saba Movaffagh ${ }^{2}$, Shohreh Alipour ${ }^{2,3 *}$ \\ ${ }^{1}$ Department of Pharmaceutics, School of Pharmacy, Shiraz University of Medical Sciences, Shiraz, Iran. \\ 2Department of Quality Control, School of Pharmacy, Shiraz University of Medical Sciences, Shiraz, Iran. \\ ${ }^{3}$ Pharmaceutical Sciences Research Center, Shiraz University of Medical Sciences, Shiraz, Iran.
}

\begin{tabular}{l}
\hline ARTICLE INFO \\
\hline Received on: 18/04/2021 \\
Accepted on: 29/06/2021 \\
Available Online: 03/11/2021
\end{tabular}

\section{Key words:}

Midazolam, intranasal delivery, poloxamer gel, pharmacokinetics.

\begin{abstract}
Midazolam (MDZ) is used as a sedative and anxiolytic agent in pediatric operations or dentistry procedures. Considering low solubility in physiologic $\mathrm{pH}$, commercial intravenous MDZ formulation is constructed as an acidic solution, which is unofficially used orally, rectally, buccally, and intranasally in spite of low patient compliance due to irritation, inflammation, and caustic effect at administration sites. Due to the pathological safety of our previously designed MDZ poloxamer nasal gel (MDZ-PLX-GEL), its pharmacokinetic parameters were determined and compared to MDZ aqueous dispersion (MDZ-DIS) after intranasal administration in rats. MDZ-PLX-GEL was prepared using PLX $(22 \% w / v)$, MDZ $(1 \% w / v)$, and propylene glycol $(10 \% v / v)$ in phosphate buffer $\mathrm{pH} 6$. For better comparison, the commercial intravenous solution was evaluated as well. MDZ was analyzed using a validated high-performance liquid chromatography method. Male Sprague Dawley rats were used for in vivo experiments. The most important feature of MDZ-PLX-GEL was a rapid onset (20 minutes) with a mean residence time (MRT) of 170 minutes, which means MDZ effective blood concentration (100-200 ng/ml) lasts 3 hours which could increase MDZ-PLX-GEL absolute bioavailability up to $66.5 \% \pm 4.6 \%$. It was confirmed that the thermosensitive poloxamer gel was a suitable nasal carrier for MDZ that could increase its residence time, which may be a proper alternative for the currently unofficial routes of MDZ administration.
\end{abstract}

\section{INTRODUCTION}

Midazolam (MDZ) is a short-acting benzodiazepine which is used as a sedative, hypnotic, and anxiolytic agent mostly in pediatric operations or diagnostic or dentistry procedures (Ashrafi et al., 2010; Bancke et al., 2015; Olivier et al., 2001; Pérez, 2012).

MDZ solubility at a physiologic $\mathrm{pH}$ is low. Therefore, the best solvent for MDZ commercial dosage form has acidic $\mathrm{pH}$

\section{${ }^{*}$ Corresponding Author}

Shohreh Alipour, Department of Quality Control, School of Pharmacy, Shiraz University of Medical Sciences, Shiraz, Iran and Pharmaceutical Sciences Research Center, Shiraz University of Medical Sciences, Shiraz, Iran.Email:alipour_sh@sums.ac.ir
3 which is unofficially administered by oral, rectal, parenteral, and intranasal routes due to the lack of a suitable commercial dosage form which resulted in different problems (Ahmad et al., 2020; Glauser et al., 2016; Shinichiro et al., 1981). Parenteral administration is usually time-consuming and needs trained personnel and shows cardiorespiratory side effects in addition to pain and irritation at the injection site. In rectal administration, besides rectal mucosa burning, variable plasma concentrations were reported which led to treatment failure. Oral use showed low bioavailability (between $35 \%$ and $44 \%$ ) in addition to the caustic and bitter taste due to $\mathrm{MDZ}$ acidic $\mathrm{pH}$. Intranasal administration showed more patient compliance, especially in children. However, nasal mucosa burning was reported (Allonen et al., 1981; Basu, 2009; Haschke et al., 2010; Henry et al., 1998; Huang et al., 1985; Marçon et al., 2009; Verma et al., 2012). 
Thus, it seems that designing a drug delivery system at a nasal physiological $\mathrm{pH}$ to decrease MDZ harmful side effects is necessary; however, a physiological $\mathrm{pH}$ will reduce MDZ solubility (Basu and Bandyopadhyay, 2011; Ilvento et al., 2015).

The highly vascularized nasal mucosa with rapid drug absorption and delivery of drugs to blood and cerebrospinal fluids makes nasal delivery an alternative route of administration (Sharma et al., 2014; Xu et al., 2014). More patient acceptability of nasal versus parenteral administration makes the intranasal route a preferred treatment, especially in children (Allonen et al., 1981; Harbord et al., 2004; Verma et al., 2012).

Low administered dose volume and high drug content are essential considerations for nasal drug delivery design. Mixed solvency is a practical approach to enhance drug solubility since it may affect the bioavailability of the administrated drug (Agrawal and Maheshwari, 2011). Therefore, MDZ-PLX-GEL was formulated in an aqueous solution containing propylene glycol (PG) in order to enhance MDZ solubility (Parhizkar et al., 2017a; Parhizkar et al., 2017b). Poloxamer 407 (Pluronic ${ }^{\circledR}$ F127) is introduced as a subject of interest for use in different formulations due to its ability to produce thermodynamically stable selfassembled structures and its good solubilizing capacity. Another important characteristic of poloxamer molecules is low toxicity and the ability to form a clear solution or gel of topical, rectal, and ocular dosage forms with no irritation or sensitivity. Poloxamer 407 hydrogel's particular mucoadhesive properties increase its potential to be a suitable carrier for "nose-to-brain" delivery of active compounds due to its avoidance of mucociliary movement and creation of noncovalent bonds with mucus, prolonging the residence time of the formulation at the application site (Giuliano et al., 2018). Poloxamer hydrogels can be used as delivery systems due to the unique properties of sol-gel conversion that is modulated by temperature. The thermosensitive sol-gel transition behavior in addition to its ability in drug steric stability improvement introduces poloxamer as one of the best carriers for nasal delivery systems. Better interaction with the nasal mucosa and slower drug release from gels can improve drug bioavailability. Another benefit of in situ gelling system is the ease of administration due to sol-gel properties, since it is liquid before administration and converts to a firm gel when dripped into the nasal cavity (Ahmad et al., 2020; Shelke et al., 2016). Our previous study indicated that the nasal MDZ-PLX-GEL was cilia friendly since unlike commercial acidic formulations its pathologic study showed no histopathological damage in a rat nasal mucosa model within 3 hours after administration. In addition, nasal MDZ-PLX-GEL showed a proper non-Newtonian rheological pattern, which is a necessary parameter in the formulation of in situ forming gels. PG was applied as a solubility enhancer to compensate MDZ aqueous solubility reduction induced by increasing $\mathrm{pH}$ (Parhizkar et al., 2017a; Parhizkar et al., 2017b).

Considering a review of previous studies focused on MDZ nasal administration, there was no similar study that evaluates intranasal MDZ gel and dispersion pharmacokinetics in rats, especially at nasal physiological $\mathrm{pH} 6$, which is the novelty of this research. Thus, the aim of this study was to determine the pharmacokinetic parameters of intranasal MDZ-PLX-GEL and MDZ dispersion (MDZ-DIS) at $\mathrm{pH} 6$ and compare them with the intravenous solution (MDZ-IV) in rats.

\section{MATERIALS AND METHODS}

\section{Materials}

Midazolam hydrochloride (MDZ) was obtained from Exir Pharmaceutical Company (Iran). Poloxamer (PLX) 407 [average molecular weight about 12,600 $(9,840-14,600)$ ] was purchased from Sigma-Aldrich. PG, potassium phosphate dibasic, and potassium phosphate monobasic were obtained from Merck. Piroxicam was gifted from Hakim Pharmaceutical Company (Iran). Methanol $(\mathrm{MeOH})$ and acetonitrile (ACN) of high-performance liquid chromatography (HPLC) grade were purchased from Samchun (Korea).

\section{Chromatography development}

\section{HPLC analysis method}

According to previous literature, a sensitive HPLC method with some modification was carried out to analyze MDZ. Wavelength was selected based on the maximal absorbance of MDZ as per UV spectrum. To conduct a precise experiment, different internal standards (diazepam, doxepin, and piroxicam) were examined based on proper retention time. Different mobile phase contents containing various ratios of phosphate buffer solution, $\mathrm{MeOH}$, and $\mathrm{ACN}$ were evaluated to obtain proper separation of MDZ and internal standard peaks with suitable retention time.

\section{Plasma extraction recovery}

Blank rat plasma was mixed with MDZ at low, mid, and high concentrations and the extraction process was carried out using different extraction solvents (ACN and $\mathrm{MeOH}$ ) separately and in combination with different ratios. A defined concentration of the internal standard was added to all MDZ samples. The peak area ratios (MDZ to internal standard) of the extracted samples were compared with standards in a neat solution (free from plasma matrix components) and the extraction recovery (\%) was calculated.

\section{Calibration curve validation}

An HPLC system consisting of a quaternary low-pressure pump CE 4104 equipped with a CE 4200 UV/Visible Detector (Cecil, England) and a HPLC column C18 (25 cm $\times 4.6 \mathrm{~mm}$ column) was applied. To construct the calibration curve, five different standard solutions of MDZ (39-625 ng/ml) were spiked with blank plasma in addition to a constant concentration of the internal standard and after extraction with optimized solvent, standard samples were assessed. Standard samples were prepared on three different days, while each concentration was tested in triplicate. The accuracy of the method was determined using known MDZ concentrations (100, 200 , and $400 \mathrm{ng} / \mathrm{ml}$ ) which were extracted and assessed in triplicate similarly. Analysis method sensitivity was determined by the limit of detection (LOD) and limit of quantitation (LOQ). Reproducibility and robustness of separation and extraction method were confirmed by validation parameters including specificity, linearity, accuracy, precision, and sensitivity.

\section{Intranasal MDZ formulation preparation}

Poloxamer powder was slowly added to cold phosphate buffer $\mathrm{pH} 6\left(5^{\circ} \mathrm{C}-10^{\circ} \mathrm{C}\right)$, under gentle magnetic 
stirring. Then, PG and MDZ $(1 \% w / v)$ were added and the mixture was left for 12 hours at $4{ }^{\circ} \mathrm{C}$ for better hydration. MDZPLX-GEL was in solution form at room temperature, whereas after nasal administration it would convert to gel form. MDZDIS was prepared by dispersion of MDZ $(1 \% w / v)$ in phosphate buffer pH 6.

\section{Animal study}

Male Sprague Dawley rats $(200 \pm 20 \mathrm{~g}$ weight and 6-8 weeks) from the Center of Comparative and Experimental Medicine (Shiraz University of Medical Sciences, Shiraz, Iran) were used for in vivo experiments. All experiments were carried out according to the Principles of Laboratory Animal Care from the National Institutes of Health's guide for the care and use of laboratory animals and guidelines for laboratory animal care and use, Animal Care and Use Committee, Shiraz University of Medical Sciences. Rats were retained in a 12 hours light/dark cycle at $22^{\circ} \mathrm{C} \pm 2^{\circ} \mathrm{C}$ and used standard diet and water ad libitum. Animals were randomly assigned to three groups (four animals per group). Rats were anesthetized by ketamine $(80 \mathrm{mg} / \mathrm{kg})$ and xylazine $(10 \mathrm{mg} / \mathrm{kg})$. Jugular vein cannulation was carried out and rats received MDZ in three different dosage forms (Parhizkar et al., 2020). The first group received intranasal MDZ-DIS, the second group received nasal MDZ-PLX-GEL, and the third group received the intravenous form of MDZ at the same dose $(4 \mathrm{mg}$ / $\mathrm{kg}$ ). Nasal dosage forms were administered by a polyethylene cannula with a diameter of $0.6 \mathrm{~mm}$. Blood samples were collected at determined times and immediately centrifuged $(15,000 \mathrm{rpm})$ for 25 minutes at $4^{\circ} \mathrm{C}$ temperature. The resultant supernatant plasma was stored at $-70^{\circ} \mathrm{C}$ until analysis.

\section{Statistical analysis}

All statistical analyses of the present study were carried out using Statistical Package for the Social Sciences software 16 and Microsoft Excel 2016. The one-way analysis of variance test was used to compare the results. A $p$-value $<0.05$ showed significant differences.

\section{RESULTS AND DISCUSSION}

\section{Chromatography development}

\section{HPLC analysis method}

A precise analysis method was determined at wavelength $240 \mathrm{~nm}$. The flow rate of the mobile phase was 1.0 $\mathrm{ml} /$ minute with a run time of 10 minutes. Better separation and proper retention time were obtained using a selected mobile phase consisting of ACN : $\mathrm{MeOH}$ : phosphate buffer $0.05 \mathrm{M} \mathrm{pH}$ 4 (40: $20: 40)$. Piroxicam was selected as an internal standard.
MDZ and piroxicam retention times were 5 and 8 minutes, respectively.

\section{Plasma extraction recovery}

To improve MDZ plasma extraction recovery, various extraction solvents were examined at different volumes and ratios. Results are shown in Table 1 . ACN was the best solvent with the highest extraction recovery more than $98 \%$; therefore, it was used as the extraction solvent in the rest of the study.

\section{Calibration curve validation}

The calibration graph of MDZ in the presence of piroxicam as an internal standard in plasma was constructed in the range of 39-625 $\mathrm{ng} / \mathrm{ml}$ (Fig. 1).

The specificity of the analysis method was confirmed by the differentiation between different concentrations of MDZ and internal standard peaks in blank rat plasma. To ensure the specificity of the HPLC method, a blank plasma chromatogram was compared with plasma-extracted MDZ and piroxicam separately and in combination. As shown in Figure 2, there was no interfering peak observed at the retention time of MDZ and piroxicam. MDZ and piroxicam were separated completely and showed proper retention time with good resolution.

A linear relationship with a correlation coefficient $\left(r^{2}\right)$ of 0.9994 was obtained at the mentioned concentration range. HPLC analysis results showed $97.4 \pm 1.6,95.7 \pm 1.1$, and $96.0 \% \pm 2.8$ intraday and interday precision and accuracy, respectively. The LOQ and LOD as parameters of sensitivity were 35 and $12 \mathrm{ng} / \mathrm{ml}$ for MDZ, respectively. The data revealed a simple and validated HPLC method to separate and specify MDZ from endogenous plasma compounds.

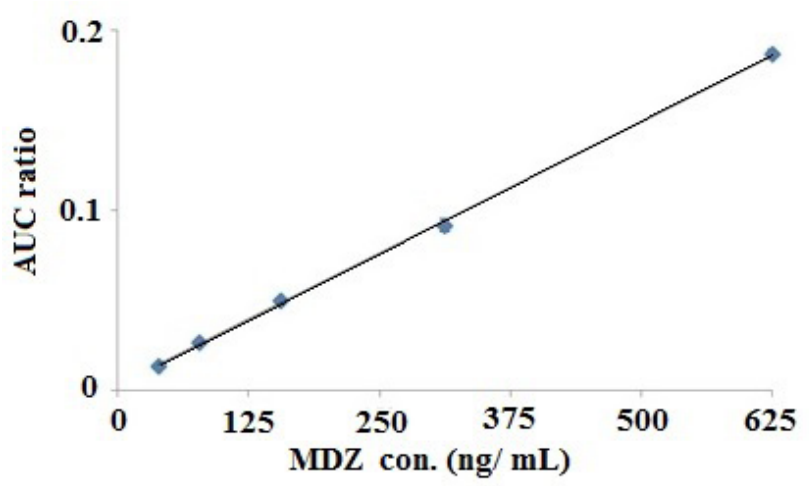

Figure 1. Calibration graph of MDZ in plasma $(n=5)$.

Table 1. Plasma extraction recovery.

\begin{tabular}{ccccccc}
\hline Solvent & MeOH & ACN & MeOH : ACN (1:1) & MeOH & ACN & MeOH : ACN (1:1) \\
\hline Solvent : plasma volume ratio & $1: 1$ & $1: 1$ & $1: 1$ & $2: 1$ & $2: 1$ & $2: 1$ \\
Recovery \% & $82.4 \pm 1.0$ & $94.1 \pm 1.1$ & $73.2 \pm 2.8$ & $86.0 \pm 1.7$ & $98.6 \pm 0.6$ & $83.2 \pm 1.1$ \\
\hline
\end{tabular}




\section{Animal study}

The concentration-time profiles of MDZ in plasma after single-dose $(4 \mathrm{mg} / \mathrm{kg})$ intravenous and intranasal administration of MDZ are shown in Figure 3. A noncompartmental model was used to estimate the main pharmacokinetic parameters (Table 2).

According to the LOD and LOQ values of the calibration curve, samples of MDZ-IV administration were quantifiable within 120 minutes after administration.

Considering previous studies, MDZ minimum therapeutic concentration in humans is $40 \mathrm{ng} / \mathrm{ml}$. The hypnotic action starts at concentrations higher than $80 \mathrm{ng} / \mathrm{ml}$, and the maximum effect was associated with higher concentrations (100$200 \mathrm{ng} / \mathrm{ml}$ ), which caused volunteers to fall asleep. However, in higher concentrations, different side effects including dizziness, muscle relaxation with ataxia, nystagmus, and slurred speech were observed (Ahmad et al., 2020; Eagleson et al., 2012; Veselis et al., 1997).

As can be seen in Figure 3, MDZ-PLX-GEL blood concentration was in the required concentration $(100-200 \mathrm{ng} / \mathrm{ml})$ within 3 hours, while the maximum concentration $\left(C_{\max }\right)$ of MDZIV was $465 \mathrm{ng} / \mathrm{ml}$ which was 4.5 times higher than the required concentration for hypnotic and sleep effects especially at the first 30 minutes and reached lower than $100 \mathrm{ng} / \mathrm{ml}$ within 2 hours which is the lowest concentration for sleeping. However, MDZDIS concentration reached a high concentration which might be harmful.

The time of reaching the maximum concentration $\left(t_{\max }\right)$ was about 20 minutes for MDZ-PLX-GEL while it was in a wide range of 5-60 minutes for MDZ-DIS, which was related to the incomplete and variable solubility of dispersed MDZ in a buffer solution at $\mathrm{pH}$ 6. The MDZ-PLX-GEL $t_{\text {max }}$ was significantly ( $p=$ 0.001 ) shorter than $t_{\max }$ in a similar study with a lower administered dose $(2 \mathrm{mg} / \mathrm{kg})$ of intranasal MDZ gel in rabbits (45-60 minutes) (Basu and Bandyopadhyay, 2011).

The mean residence time (MRT) of MDZ-PLXGEL was 170 minutes and as was predictable was significantly $(p<0.05)$ higher than MDZ-IV and MDZ-DIS (about 2.5-fold). In the previously mentioned study in rabbits, the MDZ gel MRT was 130-160 minutes, although the most important note was the duration that the most effective concentration $(100-200 \mathrm{ng} / \mathrm{mL})$ lasts which is 3 hours for MDZ-PLX-GEL, while it was reported to be 20 minutes for MDZ gel previously (Basu and Bandyopadhyay, 2011).

The absolute bioavailability of MDZ-PLX-GEL and MDZ-DIS was $66.5 \% \pm 4.6 \%$ and $60.7 \pm 38.6 \%$, respectively, which was comparable with MDZ gel's similar studies in rabbits (57-77\%); however, predictably, MDZ-DIS showed very wide variation in bioavailability (Basu and Bandyopadhyay, 2011). Intranasal MDZ gel formulation and nasal solution ( $\mathrm{pH} 3)$ in dogs showed bioavailability of $70.4 \%$ and $52.0 \%$, respectively. Previous studies mentioned that MDZ nasal formulations in acidic $\mathrm{pH}(3-4)$ caused discomfort (Eagleson et al., 2012). The effect of $\mathrm{pH}$ on nasal absorption was also studied. Higher MDZ absorption due to passive diffusion was reported at higher $\mathrm{pH}$ (above 4). Therefore, formulation of MDZ at nasal $\mathrm{pH} 6$ is necessary to enhance immediate and effective absorption (Behl et al., 1998; Giuliano et al., 2018; Harbord et al., 2004; Hardmeier et al., 2012).

This study reports the advantages of the poloxamer in situ forming gel for nasal delivery of MDZ in rats at a physiologic $\mathrm{pH}$ which has not been reported yet; however, in vivo study in rats could not explain the exact human body conditions. More studies can be performed in animals with similar nasal structures to predict MDZ pharmacokinetic characteristics in humans more accurately.
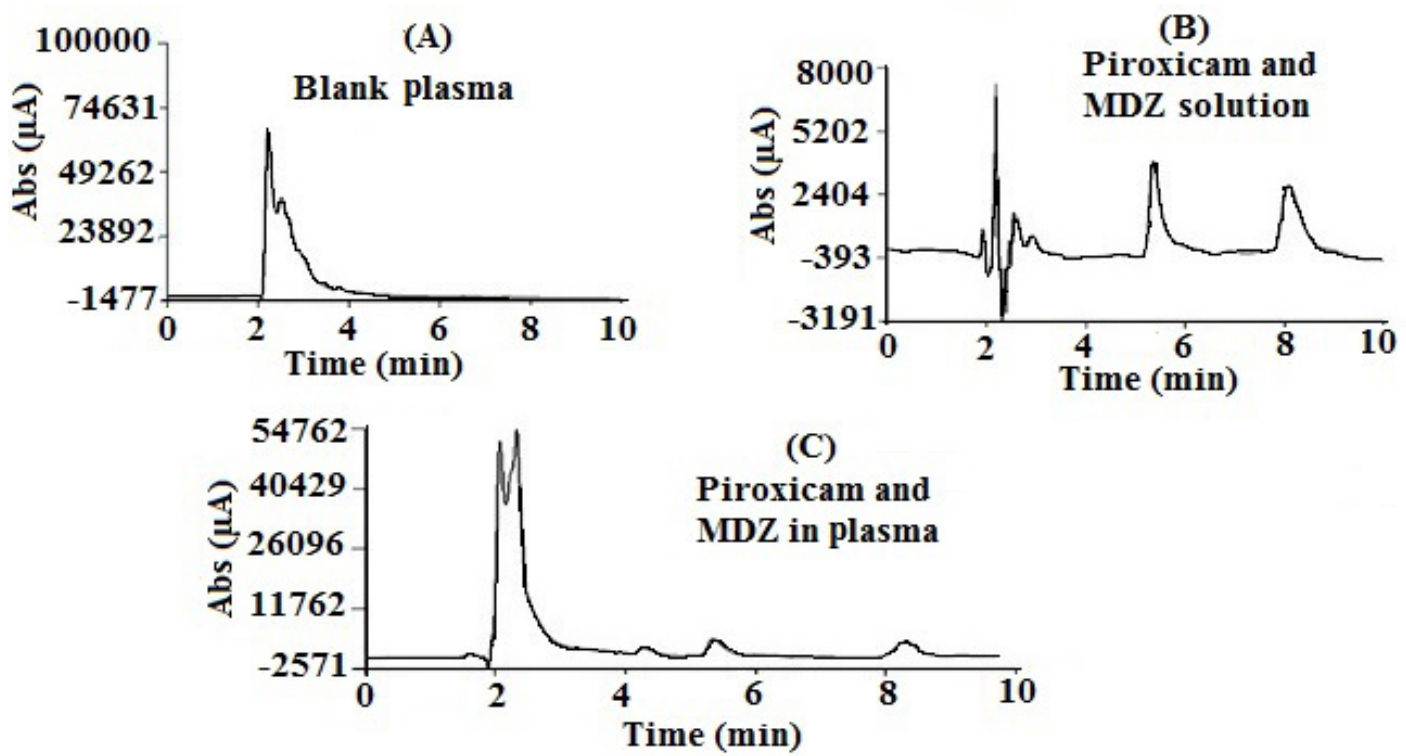

Figure 2. Chromatograms of blank plasma (A), MDZ (5 $\mu \mathrm{g} / \mathrm{ml})$ and piroxicam (1.5 $\mu \mathrm{g} / \mathrm{ml})$ solution (B), and in plasma (C). 

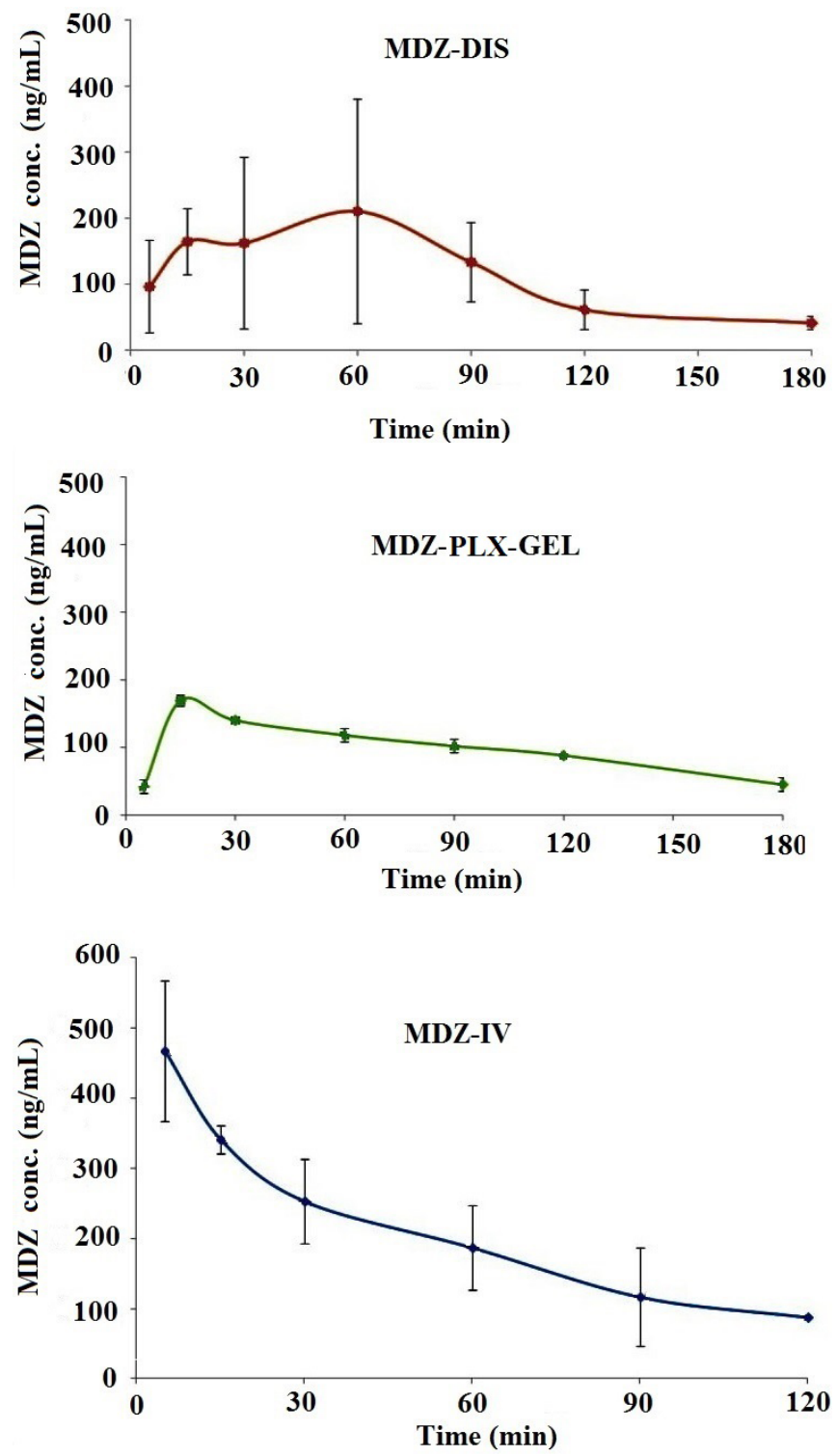

Figure 3. MDZ plasma concentration profile of nasal MDZ-DIS, nasal MDZPLX-GEL, and MDZ-IV solution in rats $(n=4)$.

\section{CONCLUSION}

The most important note in the present study was the pharmacokinetic evaluation of the MDZ-PLX-GEL formulation at nasal physiological $\mathrm{pH}$ 6. In situ nasal MDZPLX-GEL showed superior aspects against MDZ-IV and MDZDIS. Results indicated a shorter MDZ onset with a long-lasting effective concentration for MDZ-PLX-GEL. Thus, it can be concluded that intranasal thermosensitive poloxamer gel with suitable pharmacokinetics at a physiologic $\mathrm{pH}$ can be a good drug carrier for MDZ which is hoped to increase patient compliance considering its previously reported mucociliary safety.

\section{AUTHORS' CONTRIBUTIONS}

All authors made substantial contributions in design, acquisition of data, or analysis and interpretation of data; took part in drafting the manuscript; agreed to submit to the current journal; and gave final approval of the version to be published. All the authors are eligible to be authors as per the International Committee of Medical Journal Editors (ICMJE) requirements/ guidelines.

\section{ACKNOWLEDGMENTS}

This paper was extracted from a Pharm D thesis written by Saba Movaffagh and was financially supported by Shiraz University of Medical Sciences.

\section{ETHICAL APPROVAL}

All experiments were carried out according to the Principles of Laboratory Animal Care from the National Institutes of Health's guide for the care and use of laboratory animals and guidelines for laboratory animal care and use, Animal Care and Use Committee, Shiraz University of Medical Sciences.

\section{CONFLICT OF INTEREST}

The authors report no financial or any other conflicts of interest in this work.

\section{FUNDING}

This study was part of the PharmD project of Saba Movaffagh and was financially supported by Shiraz University of Medical Sciences (Grant No. 16019).

Table 2. Main pharmacokinetic parameters.

\begin{tabular}{cccc}
\hline & IV administration & \multicolumn{2}{c}{ Nasal } \\
\cline { 2 - 4 } & MDZ-IV & MDZ-PLX-GEL & MDZ-DIS \\
\hline $\boldsymbol{T}_{\max }$ (minute) & - & $20 \pm 7.1$ & $5-60$ \\
$\boldsymbol{C}_{\max }(\mathbf{n g} / \mathbf{m l})$ & $465 \pm 100$ & $169 \pm 8$ & $220 \pm 100$ \\
AUC (0- $\boldsymbol{t}$ ) $($ minutes.ng/ml) & $26,130 \pm 9,300$ & $17,382 \pm 900$ & $15,870 \pm 11,800$ \\
MRT (minute) & $52.9 \pm 6.1$ & $129.9 \pm 7.7$ & $57.1 \pm 19.2$ \\
Absolute bioavailability \% & - & $66.5 \pm 4.6$ & $60.7 \pm 38.6$ \\
\hline
\end{tabular}




\section{PUBLISHER'S NOTE}

This journal remains neutral with regard to jurisdictional claims in published institutional affiliation.

\section{REFERENCES}

Agrawal A, Maheshwari RK. Formulation development and evaluation of in situ nasal gel of poorly water soluble drug using mixed solvency concept. Asian J Pharm, 2011; 5(3):131-40.

Ahmad N, Ahmad R, Ahmad FJ, Ahmad W, Alam MA, Amir M, Ali A. Poloxamer-chitosan-based Naringenin nanoformulation used in brain targeting for the treatment of cerebral ischemia. Saudi J Biol Sci, 2020; 27(1):500-17.

Allonen H, Ziegler G, Klotz U. Midazolam kinetics. Clin Pharmacol Ther, 1981; 30(5):653-61.

Ashrafi MR, Khosroshahi N, Karimi P, Malamiri RA, Bavarian B, Zarch AV, Mirzaei M, Kompani F. Efficacy and usability of buccal midazolam in controlling acute prolonged convulsive seizures in children. Eur J Paediatr Neurol, 2010; 14(5):434-8.

Bancke LL, Dworak HA, Rodvold KA, Halvorsen MB, Gidal BE. Pharmacokinetics, pharmacodynamics, and safety of USL261, a midazolam formulation optimized for intranasal delivery, in a randomized study with healthy volunteers. Epilepsia, 2015; 56(11):1723-31.

Basu S. Development and evaluation of a mucoadhesive nasal gel of midazolam prepared with Linum usitatissimum L. Seed Mucilage Sci Pharm, 2009; 77(4):899-910.

Basu S, Bandyopadhyay AK. Characterization of mucoadhesive nasal gels containing midazolam hydrochloride prepared from Linum usitatissimum L. mucilage. Braz J Pharm, 2011; 47(4):817-23.

Behl C, Pimplaskar H, Sileno A, Romeo V. Effects of physicochemical properties and other factors on systemic nasal drug delivery. Adv Drug Deliv Rev, 1998; 29(1):089-116.

Eagleson JS, Platt SR, Strong DLE, Kent M, Freeman AC, Nghiem PP, Zheng BW, Catherine A. Bioavailability of a novel midazolam gel after intranasal administration in dogs. Am J Vet Res, 2012;73(4):539-45.

Giuliano E, Paolino D, Fresta M, Cosco D. Mucosal applications of poloxamer 407-based hydrogels: an overview. Pharmaceutics, 2018; 10(3):159-85

Glauser T, Shinnar S, Gloss D, Alldredge B, Arya R, Bainbridge J, Bare M, Bleck T, Dodson WE, Garrity L, Jagoda A, Lowenstein D, Pellock J, Riviello J, Sloan E, Treima, DM. Evidence-based guideline: treatment of convulsive status epilepticus in children and adults: report of the guideline committee of the American epilepsy society. Epilepsy Curr, 2016; 16(1):048-61.

Harbord MG, Kyrkou NE, Kyrkou MR, Kay D, Coulthard KP. Use of intranasal midazolam to treat acute seizures in paediatric community settings. J Paediatr Child Health, 2004; 40(9-10):556-8.

Hardmeier M, Zimmermann R, Ruegg S, Pfluger M, Deuster S, Suter K, Donzelli M, Drewe J, Krahenbuhl S, Fuhr P, Haschke, M. Intranasal midazolam: pharmacokinetics and pharmacodynamics assessed by quantitative EEG in healthy volunteers. Clin Pharmacol Ther, 2012; 91(5):856-62.

Haschke M, Suter K, Hofmann S, Witschi R, Fröhlich J, Imanidis G, Drewe J, Briellmann TA, Dussy FE, Krähenbühl S. Pharmacokinetics and pharmacodynamics of nasally delivered midazolam. Br J Clin Pharmacol, 2010; 69(6):607-16.

Henry RJ, Ruano N, Casto D, Wolf RH. A pharmacokinetic study of midazolam in dogs: nasal drop vs. atomizer administration. Pediatr Dent, 1998; 20(5):321-6.

Huang CH, Kimura R, Nassar RB, Hussain A. Mechanism of nasal absorption of drugs I: Physicochemical parameters influencing the rate of in situ nasal absorption of drugs in rats. J Pharm Sci, 1985; 74(6):608-11.
Ilvento L, Rosati A, Marini C, L'Erario M, Mirabile L, Guerrini R. Ketamine in refractory convulsive status epilepticus in children avoids endotracheal intubation. Epilepsy Behav, 2015; 49:343-6.

Marçon F, Mathiron D, Pilard S, Lemaire-Hurtel AS, Dubaele JM, Djedaini-Pilard F. Development and formulation of a $0.2 \%$ oral solution of midazolam containing $\gamma$-cyclodextrin. Int J Pharm, 2009; 379(2):244-50.

Olivier JC, Djilani M, Fahmy S, Couet W. In situ nasal absorption of midazolam in rats. Int J Pharm, 2001; 213(1-2):187-92.

Parhizkar E, Emadi L, Alipour S. Development and evaluation of midazolam in situ nasal gel properties in presence of solubility enhancers at cilia-friendly $\mathrm{pH}$. Macromol Res, 2017a; 25(3):255-61.

Parhizkar E, Emadi L, Alipour S. Midazolam solubility enhancement pre-formulation techniques at nasal physiological $\mathrm{pH}$. Int J Pharm Sci Res, 2017b; 8(5):2315-22.

Parhizkar E, Mohammadi Z, Alipour S. Rapid high-performance liquid chromatography method for levodopa quantitation at low UV wavelength: application of pharmacokinetics study in rat following intranasal delivery. J Chromatogr Sci, 2020; 59(4):347-54.

Pérez C. Development and validation of a method to quantify midazolam in a new oral formulation for pediatric use. Am J Anal Chem, $2012 ; 3(8): 552-8$.

Sharma S, Lohan S, Murthy R. Formulation and characterization of intranasal mucoadhesive nanoparticulates and thermo-reversible gel of levodopa for brain delivery. Drug Dev Ind Pharm, 2014; 40(7):869-78.

Shelke S, Shahi S, Jalalpure S, Dhamecha D. Poloxamer 407-based intranasal thermoreversible gel of zolmitriptan-loaded nanoethosomes: formulation, optimization, evaluation and permeation studies. J Liposome Res, 2016; 26(4):313-23.

Shinichiro H, Takatsuka Y, Tai M, Hiroyuki M. Absorption of drugs from the nasal mucosa of rat. Int J Pharm, 1981; 7(4):317-25.

Verma RK, Paswan A, De A, Gupta S. Premedication with midazolam nasal spray: an alternative to oral midazolam in children. Anesth Pain Med, 2012; 1(4):248-55.

Veselis RA, Reinsel RA, Feshchenko VA, Wronski M. The comparative amnestic effects of midazolam, propofol, thiopental, and fentanyl at equisedative concentrations. Anesthesiology, 1997; 87(4):749-64.

Xu X, Shen Y, Wang W, Sun C, Li C, Xiong Y, Tu J. Preparation and in vitro characterization of thermosensitive and mucoadhesive hydrogels for nasal delivery of phenylephrine hydrochloride. Eur J Pharm Biopharm, 2014; 88(3):998-1004.

How to cite this article:

Parhizkar E, Movaffagh S, Alipour S. Pharmacokinetics and bioavailability of midazolam in rats following single dose administration of intravenous solution, intranasal dispersion, and in situ nasal gel. J Appl Pharm Sci, 2021; 11(11):070-075. 